\title{
The adverse effects of cannabinoids: implications for use of medical marijuana
}

\author{
Louisa Degenhardt MPsych(Clin) PhD, Wayne D. Hall PhD
}

$\infty$

See related article page 1669

$\mathrm{C}$ annabis preparations have been used to relieve nausea, improve appetite and reduce pain for thousands of years. ${ }^{1}$ The development of synthetic drugs in the 2oth century supplanted these and other herbal remedies, but in the past several decades there has been a resurgence of interest in using cannabis and cannabinoid preparations for medical purposes. There is evidence from controlled trials that cannabinoids are effective in relieving nausea and vomiting, alleviating acute pain and improving appetite in people with HIV-related disorders. ${ }^{1}$

A number of synthetic cannabinoids (e.g., dronabinol) have been approved for medical use in Canada, the United Kingdom and the United States. More recently, a cannabis extract (Sativex) has been provisionally approved in Canada for the treatment of multiple sclerosis. Not all patients have found that the drugs relieve their symptoms, primarily because they find it difficult to control the desired dose of orally administered cannabinoids. ${ }^{2,3}$ In 200I, the Canadian government responded to demands to allow medical use of smoked cannabis, if authorized by a doctor, among patients experiencing severe symptoms related to cancer, HIV infection, multiple sclerosis and epilepsy. ${ }^{4}$ Symptoms include nausea, pain, weight loss and persistent muscle spasms and seizures. This decision was made in the absence of strong evidence supporting the efficacy of cannabinoids in many of these applications.

Most importantly, the decision was made in the absence of evidence on the safety of smoking cannabis for medical purposes. This is a problem because the Marihuana Medical Access Regulations require that a doctor discuss the risks of medical cannabis use with his or her patient. ${ }^{4}$ In the absence of information on the risks of medical cannabis use, some critics have noted that it is difficult for doctors to comply with this provision of the regulations. ${ }^{5}$

In the current issue of $C M A J$, Wang and colleagues ${ }^{6}$ present a systematic review of the research on adverse effects of medical cannabis use. They included 23 randomized controlled trials and 8 observational studies of cannabinoids and cannabis extracts for various medical indications.

The authors found that most of the adverse events $(96.6 \%$ [4615/4779]) reported in the randomized clinical trials were not serious; dizziness was the most common of these (15.5\% [714/4615]). They found that, although the rate of nonserious adverse events was higher among participants assigned to medical cannabinoids than among controls, the rates of serious adverse events did not differ significantly between the 2 groups (rate ratio I.04, 95\% confidence interval 0.78-I.39). ${ }^{6}$

\section{Key points}

- Smoking cannabis for medical purposes has been approved in the absence of strong evidence of its safety and efficacy.

- The current review of evidence from clinical trials of cannabinoids and cannabis extracts for medical indications suggests that the adverse effects of their short-term use are modest.

- Research is needed to evaluate the adverse effects of longterm use of medical cannabinoids, such as for the treatment of multiple sclerosis and chronic pain.

These findings support the conclusions of the Institute of Medicine, ${ }^{1}$ that short-term use of cannabinoids for medical purposes has an acceptable safety profile. This provides reassurance on the safety of prescribing cannabinoids and the newer cannabis extract for the short-term symptomatic relief of nausea and vomiting in cancer treatment and for the relief of acute pain (e.g., postoperatively). However, the review by Wang and colleagues is unable to provide information on the long-term use of cannabinoids for chronic disorders, such as multiple sclerosis, because the available trials were of relatively short duration (8 hours to 12 months). ${ }^{6}$

None of the trials involved the smoking of cannabis. Observational population-based cohort studies of recreational cannabis users provide some indication of what the adverse events of long-term cannabis smoking may be. These studies have largely examined the effects in adolescence and early adulthood of sustained recreational use of cannabis among young people. ${ }^{7}$ More rarely, they have examined rates of long-term adverse health effects, such as cancer, that may arise from prolonged exposure to carcinogens contained in cannabis smoke. ${ }^{8,9}$ It is not clear whether medical use of cannabis among older adults would lead to the psychosocial outcomes that occur in younger adults. The consequences that are of most concern to clinicians and patients are the risks of developing cannabis dependence, exacerbation of cardiovascular disease, precipitation of psychotic disorders and cancers.

Cannabis dependence can develop, ${ }^{10}$ but the evidence on risk factors for dependence comes primarily from studies

Louisa Degenhardt and Wayne Hall are with the National Drug and Alcohol Research Centre, University of New South Wales, Sydney, Australia. Wayne Hall is also with the School of Population Health, University of Queensland, Brisbane, Australia. 
involving recreational users who began using cannabis in adolescence and early adulthood and who use the most potent products. These users smoke cannabis with a greater frequency and intensity than older adults who would use smaller doses for symptom relief. ${ }^{11}$ We know nothing of the risks of incident cannabis dependence in the context of long-term, supervised medical use.

There has been concern about an association between cannabis use and psychosis, with evidence suggesting that cannabis may precipitate psychotic symptoms or disorders in people vulnerable to such symptoms. ${ }^{12}$ However, this evidence also comes from studies involving recreational users. There are currently no data on the extent of risk for psychotic symptoms among medical users, but those who are vulnerable to psychosis because of personal or family history would be ill-advised to use cannabis heavily for any reason. ${ }^{12,13}$

The risk of cardiovascular disease is potentially more concerning given that the population using cannabis for medical purposes probably has a higher prevalence of cardiovascular problems. ${ }^{14}$ There is little evidence on the extent of risk for adverse cardiovascular outcomes among cannabis users with pre-existing conditions. In a recent exploratory study, Mukamal and colleagues ${ }^{15}$ found that people who used cannabis heavily (in addition to heavy use of tobacco and alcohol) and who had other elevated risk factors for adverse outcomes were at slightly elevated risk for death at later follow-up, but deaths were not related to cardiovascular causes. More research on this topic is needed. The risk factors for cancer associated with cannabis smoking remain uncertain given inconsistent findings: some studies have suggested no increased risk, whereas case-control studies have suggested some elevation of risk with heavy long-term use. ${ }^{8,9}$

The findings from Wang and colleagues' review suggest that, based on the current data, the risk of adverse events associated with short-term medical use of cannabinoids and cannabis extracts are minor. The risk seems to be marginally greater with oral use of $\Delta$-9-tetrahydrocannabinol than with oral use of combined $\Delta$ - 9 -tetrahydrocannabinol-cannabidiol. ${ }^{6}$ These differences matter because the cannabis plant contains hundreds of cannabinoids, and cannabidiol may counteract some of the adverse effects of $\Delta$-9-tetrahydrocannabinol. ${ }^{16}$ We need better information on the adverse effects of more sustained use of cannabinoids and cannabis preparations from follow-up studies involving patients who use these preparations.

There is a more pressing need for follow-up studies involving patients who smoke cannabis for medical purposes, to assess the risk for cannabis dependence and cardiovascular disease. These studies should involve Canadian patients au- thorized to use herbal cannabis (226I as of October $2007^{6}$ ), who have been willing to do so despite their doctors' limited capacity to provide detailed information on the magnitude of risks associated with such use.

Competing interests: None declared.

Contributors: Both of the authors contributed to the conception and design of the manuscript, drafted and revised the article and approved the final version for publication.

Acknowledgements: The National Drug and Alcohol Research Centre is funded by the Australian Government Department of Health and Ageing. Louisa Degenhardt is the recipient of an Australian National Health and Medical Research Council Senior Research Fellowship. In 2000-200I, Wayne Hall was the Chair of the Working Party on the Use of Cannabis for Medical Purposes in New South Wales, Australia. Louisa Degenhardt was the Research Officer for the Working Group.

\section{REFERENCES}

I. Institute of Medicine. Marijuana and medicine: assessing the science base. Washington: National Academy Press; 1999.

2. Working Party on the Use of Cannabis for Medical Purposes. Report of the Working Party on the Use of Cannabis for Medical Purposes. Volume II. Sydney (AU): National Drug and Alcohol Research Centre, University of NSW; 2000.

3. O'Connell TJ, Bou-Matar CB. Long term marijuana users seeking medical cannanbis in California (200I-2007): demographics, social characteristcis, patterns of cannabis and other drug use of 4II7 applicants. Harm Reduct J 2007;4:I6.

4. Marihuana Medical Access Regulations. SOR/200I-227. Available: http://laws.justice.gc.ca/en/showtdm/cr/SOR-200I-227 (accessed 2008 Apr 6).

5. Kalant H. Smoked marijuana as medicine: not much future. Clin Pharmaco Ther 2008;83:517-9.

6. Wang T, Collet JP, Shapiro S, et al. Adverse effects of medical cannabinoids: a systematic review. CMAJ 2008;178:1669-78.

7. Moore TH, Zammit S, Lingford-Hughes A, et al. Cannabis use and risk of psychotic or affective mental health outcomes: a systematic review. Lancet 2007;370:319-28.

8. Hashibe M, Morgenstern $\mathrm{H}$, Cui $\mathrm{Y}$, et al. Marijuana use and the risk of lung and upper aerodigestive tract cancers: results of a population-based case-control study. Cancer Epidemiol Biomarkers Prev 2006;15:1829-34.

9. Aldington S, Harwood M, Cox B, et al. Cannabis use and risk of lung cancer: case-control study. Eur Respir J 2008;31:280-6.

Io. Hall W, Degenhardt L, Lynskey M. The health and psychological consequences of cannabis use. Canberra (AU): Australian Publishing Service, 200I.

II. Hall WD, Swift W. The policy implications of cannabis dependence. In: Roffman RA, Stephens RS, editors. Cannabis dependence: its nature, consequences and treatment. Cambridge University Press (UK): 2006. p. 315-39.

I2. Degenhardt L, Hall W. Is cannabis a contributory cause of psychosis? Can J Psychiatry 2006;51:556-65

I3. Hall W, Degenhardt L. What are the policy implications of the evidence on cannabis and psychosis? Can J Psychiatry 2006;51:566-74.

I4. Hall W, Pacula RL. Cannabis use and dependence: public health and the public policy. London (UK): Cambridge University Press; 2003.

I5. Mukamal KJ, Maclure M, Muller J, et al. Acute ischemic heart disease: an exploratory prospective study of marijuana use and mortality following acute myocaridal infarction. Am Heart J 2008; 155:465-70.

I6. Zuardi AW, Crippa JA, Hallak JE, et al. Cannabidiol, a cannabis sativa constituent, as an antipsychotic drug. Braz J Med Biol Res 2006;39:42I-9.

Correspondence to: Dr. Louisa Degenhardt, Professor of Epidemiology, National Drug and Alcohol Research Centre, University of New South Wales, Sydney NSW 2052, Australia; fax 6I 29385 0222; l.degenhardt@unsw.edu.au 